\title{
Comprehensive Analysis of miRNA-mRNA- IncRNA Networks in Non-Smoking and Smoking Patients with Chronic Obstructive Pulmonary Disease
}

\author{
Yan Qian Zheng-dao Mao Yu-jia Shi Zhi-guang Liu Qi Cao Qian Zhang \\ Department of Respiratory and Critical Care Medicine, The Affiliated Changzhou No. 2 People's \\ Hospital of Nanjing Medical University, Changzhou, China
}

\section{Key Words}

IncRNAs • miRNAs • genes • smoking • Chronic Obstructive Pulmonary Disease (COPD)

\begin{abstract}
Background/Aims: Chronic obstructive pulmonary disease (COPD) is a major cause of morbidity and mortality worldwide. This study aimed to identify overlapping or diverging dysregulated genes, IncRNAs, miRNAs and signaling pathways in smoking and non-smoking chronic obstructive pulmonary disease (COPD). Methods: Compared to normal controls, we identified the shared and divergent differentially expressed mRNAs (DEmRNAs), miRNAs (DEmiRNAs) and IncRNAs (DElncRNAs) in smoking and non-smoking COPD by RNAsequencing and bioinformatics analysis. Functional annotation of DEmRNAs were performed. Both cis and trans-target DEmRNAs of DEIncRNAs were identified. The target DEmRNAs of DEmiRNAs were identified as well. The DEmiRNA-DEmRNA-DElncRNA interaction network was constructed. QRT-PCR was performed to validat the selected DEmiRNAs, DEmRNA and DElncRNAs in COPD. Results: Compared to normal control, 1234 DEmRNAs, 96 DElncRNAs and 151 DEmiRNAs were identified in non-smoking patients with COPD; 670 DEmRNAs, 44 DElncRNAs and 63 DEmiRNAs were identified in smoking patients with COPD. Leukocyte transendothelial migration and pathways in cancer were significantly enriched pathways in non-smoking and smoking COPD, respectively. MiR-122-5p-A2M-LINC00987/A2M-AS1/ linc0061 interactions might play key roles in COPD irrespective with the smoking status. Let7-ADRB1-HLA-DQB1-AS1 might play a key role in the pathogenesis of smoking COPD while miR-218-5p/miR15a-RORA-LOC101928100/LINC00861 and miR-218-5p/miR15a-TGFß3RORA-AS1 interactions might involve with non-smoking COPD. Conclusion: We identified the shared and diverging genes, IncRNAs, miRNAs and their interactions and pathways in smoking and non-smoking COPD which provided clues for understanding the mechanism and developing novel diagnostic and therapeutic strategies for COPD.

Y. Qian and Z. Mao contributed equally to this work. $\begin{array}{ll}\text { Qian Zhang } & \begin{array}{l}\text { Dept. of Respiratory and Critical Care Med, The Affiliated Changzhou No. } 2 \text { People's Hospital } \\ \text { of Nanjing Medical University, } 29 \text { Xinlong Road, Changzhou } 213003 \text { (China) }\end{array}\end{array}$ Tel. 86-0519-81087391, Fax 86-0519-88115560, E-Mail zhang_qiannj@163.com
\end{abstract}




\section{Cellular Physiology Cell Physiol Biochem 2018;50:1140-1153

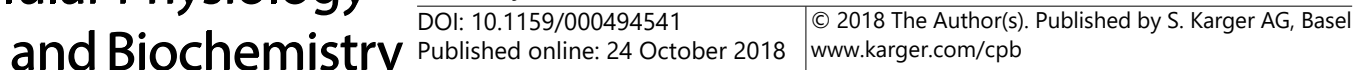 \\ Qian et al.: miRNA-mRNA-IncRNA Networks in COPD}

\section{Introduction}

Chronic obstructive pulmonary disease (COPD) is a progressive disease that characterized by the presence of chronic bronchitis or emphysema which may result in the development of airway obstruction [1]. As a major global disease, COPD has been predicted to be the third leading cause of mortality worldwide by the year 2020[2]. COPD is a multifactorial complex disease that was associated with the interactions of genetic and environmental risk factors while its pathogenesis remains largely unknow [3].

Although cigarette smoking has long been widely known as the single most important risk factor for COPD [3], more than 25\% patients with COPD were non-smokers [4-6]. However, previous studies mainly focused on COPD in smokers, studies of non-smoking COPD were limited [7]. The mechanism of COPD, especially in never-smokers, and the biomarkers and therapeutic targets of this disease at molecular levels have become targets of interest.

Besides key genes involved in the pathogenesis of COPD, non-coding RNAs including microRNAs (miRNAs) and long noncoding RNAs (lncRNAs) were reported to involve with COPD $[8,9]$. MiRNAs are a class of small endogenous ncRNAs with 19-25 nucleotides that negatively regulate gene expression at the post-transcriptional level $[8,10]$. LncRNAs are a class of ncRNAs that more than 200 nucleotides which could regulate the expression of protein-coding genes by cis- or trans-regulatory effects [11]. LncRNAs have been demonstrated to involve in various physiological and pathological processes [12].

In this study, we aimed to identify overlapping or diverging genes, IncRNAs, miRNAs and signaling pathways in smoking and non-smoking COPD. Compared to normal controls, the differentially expressed mRNAs (DEmRNAs), miRNAs (DEmiRNAs) and IncRNAs (DElncRNAs) in non-smoking COPD and smoking COPD were identified, respectively. Based functional annotation and bioinformatics analysis, the shared and divergent DEmiRNADEmRNA-DElncRNA interactions in non-smoking and smoking COPD were further identified which provided clues for understanding the mechanism and developing novel diagnostic and therapeutic strategies for COPD.

\section{Materials and Methods}

\section{Patients and samples}

Five smoking patients with COPD (S1-S5), five non-smoking patients with COPD (N1-N5) and five normal controls (C1-C5) were enrolled in this study from the Affiliated Changzhou No. 2 People's Hospital with Nanjing Medical University. The detailed information of all these 15 participants were displayed in Table 1. Since smoking patients with COPD in this hospital were all males, gender difference existed between smoking COPD group and normal controls in this study. However, different susceptibility of COPD between genders is believed to result from the different incidence of smoking between genders rather than to any gender driven susceptibility $[13,14]$. Hence, we speculated that gender difference has little effect on our results. Due to restrictions in sample collection, significant differences existed between non-smoking patients with COPD and normal controls $(p<0.05)$ as well as between smoking patients with COPD and normal controls $(p<0.05)$. The blood samples were obtained from all these 15 participants. This study was approved by the Ethics Committee of The Affiliated Changzhou No.2 People's Hospital with Nanjing Medical University. The signed informed consent of all these participants was obtained. This research complied with the principles of the Declaration of Helsinki.

\section{Library preparation and high-throughput sequencing}

Total RNA was extracted from blood samples by using by TRIzol reagent (Invitrogen, Carlsbad, CA, USA) according to the manual instruction. Nanodrop ND-2000 spectrophotometer (Thermo Scientific, Wilmington, DE, USA) was used to check the concentration and purity of RNA. The integrity of RNA was checked by using 2\% agarose gel. Agilent 2100 Bioanalyzer (Agilent Technologies, Palo Alto, Calif.) was used to obtain the RIN value. RNA with amount $>5 \mu \mathrm{g}$, concentration $\geq 200 \mathrm{ng} / \mathrm{ml}, 1.8<0 D 260 / 280<2.2$ and RIN $>7$ was used to prepare cDNA library construction.

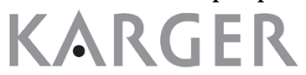




\section{Cellular Physiology Cell Physiol Biochem 2018;50:1140-1153

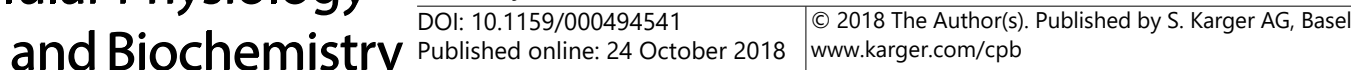 \\ Qian et al.: miRNA-mRNA-IncRNA Networks in COPD}

Firstly, we constructed the library for high throughput sequencing of IncRNA and mRNA as follow steps: 1). Remove the ribosomal RNA from total RNA by Ribo-Zero Magnetic kit (EpiCentre, Mandison, WI, USA); 2). Construct the library with Truseq RNA sample Prep Kit (Illumina, Inc., San Diego, CA, USA). RNA was purified and fragmented into 200 500 base pairs; the first cDNA strand was synthesized by RNA fragments primed with random hexamer primers; the second cDNA strand was synthesized with dUTP instead of dTTP; after purification by Qiaquick PCR purification kit, end repair, 3'end adenylation and adapter ligation were performed; the second cDNA strand was digested by using UNG enzyme (Illumina, Inc., San Diego, CA, USA) and polymerase chain reaction (PCR) was performed to construct library for high throughput sequencing of IncRNA and mRNA.

Secondly, we isolated the 18-30 nt RNA from the total RNA and performed adapter ligation and RT-PCR to construct the cDNA library for high throughput sequencing of miRNA by TruseqTM Small RNA sample prep Kit.

Thirdly, all libraries for high throughput sequencing of IncRNA and mRNA were amplified through PCR for 15 cycles. Then, purification was performed by Certified Low Range Ultra Agarose (Bio-Rad) and quantification was performed by Picogreen (Molecular probes) on TBS380 (Turner Biosystems). Bridge PCR was performed on cBot. Sequencing was performed by using a llumina HiSeq X Ten platform.

Lastly, all libraries for high throughput sequencing of miRNA were amplified through PCR for 12 cycles. Then, purification was performed by $6 \%$ Novex TBE PAGE gel (1.0 mm, 10 well) and quantification was performed by Picogreen (Molecular probes) on TBS380 (Turner Biosystems). Bridge PCR was performed on cBot. Sequencing was performed by a llumina HiSeq 4000 platform.

Quality control of raw sequence

By using Base Calling, all the raw data obtained from high-throughput RNA-sequencing was translated into raw FASTQ sequence data. To obtain the clean reads, sequence with low quality including adaptor sequences, sequences with quality score $<20$ and sequences with $\mathrm{N}$ base rate of raw reads $>10 \%$ were removed by using cutadapt (http://cufflinks.cbcb.umd.edu/).

\section{Clean reads mapping}

By using TopHat (http://ccb.jhu.edu/software/tophat/index.shtml), clean reads from RNA-sequencing results of mRNA and IncRNA were aligned with the human reference genome, Ensembl V84. By using Cufflinks (http://cufflinks.cbcb.umd.edu/), the expression of lncRNA and mRNA was quantified and the normalized expression data were outputted. By using bowtie (http://bowtie-bio.sourceforge.net/index. shtml), clean reads from RNA-sequencing results of miRNA were aligned with the human reference genome, GRCH38. The expression of miRNA was quantified by using miRDeep2.

\section{Identification of DEmRNAs, DEmiRNAs and DEIncRNAs}

Compared to normal controls, DEmRNAs, DEmiRNAs and DElncRNAs in smoking patients with COPD and non-smoking patients with COPD were identified by DEGseq (http://bioconductor.org/packages/ DEGseq/), respectively. FDR $<0.01$ and $\mid \log 2$ Fold Change $\mid>1$ were selected as the criteria of significance.

\section{Functional analysis of DEmRNAs}

Gene ontology (GO) and Kyoto Encyclopedia of Genes and Genomes (KEGG) molecular pathway enrichment analysis of DEmRNAs in smoking and non-smoking patients with COPD were performed by using online-based software GeneCoDis3 (http://genecodis.cnb.csic.es/analysis), respectively.

\section{DEmRNA-DEIncRNA interaction analysis}

LncRNAs were reported to regulate genes that transcribed near them, consistent with activity in cis [15]. To identify the nearby DEmRNAs of DElncRNAs with cis-regulatory effects, DEmRNAs transcribed within a $100 \mathrm{~kb}$ window up- or down-stream of DElncRNAs in smoking and non-smoking patients with COPD were searched, respectively.

In addition, DEmRNAs co-expressed with DElncRNAs were identified as well. We calculated the pairwise Pearson correlation coefficients between DElncRNAs and DEmRNAs in smoking and non-smoking patients with COPD, respectively. DElncRNA-DEmRNA pairs with PCC $>0.9$ were served as significant 


\section{Cellular Physiology Cell Physiol Biochem 2018;50:1140-1153 \begin{tabular}{l|l|l} 
DOI: 10.1159/000494541 & $\begin{array}{l}\text { O } 2018 \text { The Author(s). Published by S. Karger AG, Basel } \\
\text { www.karger.com/cpb }\end{array}$
\end{tabular} \\ Qian et al.: miRNA-mRNA-IncRNA Networks in COPD}

DElncRNA-DEmRNA co-expression pairs. The DElncRNA-nearby target DEmRNAs pairs and DEIncRNA-DEmRNA co-expression pairs were used to construct the DEIncRNA-DEmRNA interaction network by using Cytoscape software (http://www.cytoscape.org/).

DEmiRNA-DEmRNA interaction analysis

Firstly, the pairwise Pearson correlation coefficients between DEmRNAs and DEmiRNAs were calculated. DEmiRNA-DEmRNA pairs with $\mathrm{p}<0.05$ and $\mathrm{r}<0$ were served as significant negative DEmiRNA-DEmRNA co-expression pairs. Then, we obtained the putative target DEmRNAs of DEmiRNAs by six bioinformatic algorithms (RNA22, miRanda, miRDB, miRWalk, PICTAR2 and Targetscan). Moreover, the confirmed target mRNAs of miRNAs were obtained by miRWalk. Among these obtained significant negative DEmiRNADEmRNA co-expression pairs, the confirmed DEmiRNA-DEmRNA pairs by miRWalk and the DEmiRNA-DEmRNA pairs recorded by $\geq 4$ algorithms were served as target DEmRNAs of DEmiRNAs in our study.

\section{DEmiRNA-DEmRNA-DEIncRNA interaction network}

Based on the DElncRNA-DEmRNA interaction analysis and DEmiRNADEmRNA interaction analysis, we identified the DEmRNAs which were interacted with both DElncRNAs and DEmiRNAs and constructed the DEmiRNA-DEmRNA-DElncRNA interaction network.

\section{Confirmation by $q R T-P C R$}

To verify the expression of DEmRNAs, DEmiRNAs and DElncRNAs of our RNA-sequencing results, we collected 20 blood samples from 20 individuals including 7 smoking patients with COPD (S1-S7) and 7 non-smoking patients with COPD (N1-N7) and 6 normal controls (C1-C6). Fifteen out of these 20 individuals (S1-S5, N1-N7 and C1-C5) were whom enrolled for RNAsequencing as well. The detailed information of other five individuals (S6, S7, N6, N7 and C6) were displayed in Table 1.

Total RNA was isolated by using the Trizol reagent (Invitrogen, USA) according to manufacturer's protocol. The mRNA template was reversely transcribed into cDNA using a FastQuant RT Kit according to the manufacturer's protocol. MiRNA reverse transcription was performed using miRcute miRNA First-strand cDNA Synthesis kits (TIAN- GEN) according to the manufacturer's instructions. The qRT-PCR reactions were performed using SuperReal PreMix Plus (Invitrogen, USA) in ABI 7500 Real-time PCR Detection System. Relative gene expression was analyzed using $2^{-\Delta \Delta C t}$ method. The human 18srRNA were used as endogenous controls for mRNA and IncRNA and human U6 were used as endogenous controls for miRNA expression in analysis. 


\section{Cellular Physiology Cell Physiol Biochem 2018;50:1140-1153 \begin{tabular}{l|l|l} 
and Biochemistry Published online: 24 October 2018 & $\begin{array}{l}\text { (c) } 2018 \text { The Author(s). Published by S. Karger AG, Basel } \\
\text { www.karger.com/cpb }\end{array}$ \\
\hline
\end{tabular} \\ Qian et al.: miRNA-mRNA-IncRNA Networks in COPD}

\section{Results}

Identification of DEmRNAs, DEmiRNAs and DEIncRNAs

Compared to normal controls, 1234 DEmRNAs (440 upregulated and 794 down-regulated DEmRNAs), 96 DElncRNAs (20 upregulated and 76 down-regulated DElncRNAs) and 151 DEmiRNAs (103 up-regulated and 48 downregulated DEmiRNAs) in nonsmoking patients with COPD were identified. The top 10 DEmRNAs, DEmiRNAs and DElncRNAs between non-smoking patients with COPD and normal controls were displayed in Table 2 .

Compared to normal control, 670 DEmRNAs (249 upregulated and 421 down-regulated DEmRNAs), 44 DElncRNAs (15 upregulated and 29 down-regulated DElncRNAs) and 63 DEmiRNAs (35 up-regulated and 28 downregulated DEmiRNAs) in smoking patients with COPD were identified. The top 10 DEmRNAs, DEmiRNAs and DElncRNAs between smoking patients with COPD and normal controls were displayed in Table 2 as well.

Moreover, 225 shared DEmRNAs (82 up-regulated and 143 down-regulated DEmRNAs), 15 shared DElncRNAs ( 3 up-regulated and 12 down-regulated DElncRNAs) and 31 shared DEmiRNAs (18 up-regulated and 13 down-regulated DEmiRNAs) were identified in both smoking and non-smoking patients with COPD compared to normal controls.

\section{Functional annotation of DEmRNAs}

Based on the functional annotation of DEmRNAs between non-smoking patients with COPD and normal controls, immune response (FDR=5.78E-17), response to external stimulus (FDR=1.10E-16), immune system process (FDR=2.75E-16), molecular transducer activity (FDR=2.19E-08) and cell periphery (FDR=8.43E-18) were significantly enriched GO terms (Table 3). Based on the KEGG enrichment analysis in non-smoking COPD (Fig. S1A for all supplemental material see www.karger.com/10.1159/000494541/), Transcriptional misregulation in cancer (FDR=2.80E-03, Fig. S1B) and pathways in cancer (FDR=1.34E-02) were significantly enriched pathways.

According to the functional annotation of DEmRNAs between smoking patients with COPD and normal controls, cellular response to chemical stimulus (FDR=5.36E-07), regulation of cell proliferation (FDR $=1.98 \mathrm{E}-05)$, cell surface receptor signaling pathway (FDR=2.21E-05), protein binding (FDR=1.17E-06) and plasma membrane (FDR=1.32E-08) were significantly enriched GO terms (Table 3\&4). Based on the KEGG enrichment analysis in smoking COPD (Fig. S1C), leukocyte transendothelial migration (FDR=4.19E-02) was a significantly enriched pathway in smoking COPD (Fig. S1D).

Moreover, cytokine-cytokine receptor interaction, ECM-receptor interaction, PI3K-Akt signaling pathway and cell adhesion molecules were four shared pathways in both smoking and non-smoking COPD.
Table 2. The top 10 DEmRNAs, DElncRNAs and DEmiRNAs in smoking and non-smoking COPD compared to normal controls. FC, fold change

\begin{tabular}{|c|c|c|c|c|c|c|}
\hline & \multicolumn{3}{|c|}{ Smoking COPD } & \multicolumn{3}{|c|}{ Non-smoking COPD } \\
\hline DEmRNA & Regulation & $\operatorname{LogFC}$ & FDR & DEmRNA & Regulation & LogFC \\
\hline IFI27 & up & 5.76 & FDR $<0.01$ & PRSS33 & down & -6.93 FDR $<0.01$ \\
\hline SP & down & -4.28 & $\mathrm{FDR}<0.01$ & XKR3 & up & 6.02 FDR $<0.01$ \\
\hline TXN2 & own & -3.95 & FDR $<0$ & IFI27 & up & $5.50 \mathrm{FDR}<\mathrm{C}$ \\
\hline ITRNR2 & down & -3.91 & FDR $<0.01$ & ALOX15 & down & $-5.03 \quad F D R<0.01$ \\
\hline CDC3 & up & 3.69 & FDR $<0.01$ & FN1 & up & $4.77 \quad$ FDR $<0.01$ \\
\hline LA-DQF & up & 3.53 & FDR $<0.01$ & SIGLEC8 & down & -4.34 FDR $<0.01$ \\
\hline YOM2 & down & -3.45 & FDR $<0$ & HDC & down & -4.29 FDR $<0.01$ \\
\hline $\mathrm{AOA}$ & up & 3.20 & FDR $<0.01$ & GBP6 & up & $3.98 \quad$ FDR $<0.01$ \\
\hline ИTC1 & up & 3.06 & FDR $<0$ & ANK & up & $3.89 \mathrm{FDR}<$ \\
\hline LCI & down & -3.05 & FDR $<0.01$ & R2 & down & -3.77 FDR $<0.01$ \\
\hline Elnch & Regulation & $\operatorname{LogFC}$ & FDR & DEln & Regulatiol & LogFC FDR \\
\hline IST & down & -10.42 & FDR $<0.01$ & FAM & up & $2.39 \mathrm{FDR}<$ \\
\hline SIX & wn & -3.87 & FDR $<$ & $\mathrm{A} 2 \mathrm{M}$ & down & -2.18 FDR $<$ \\
\hline $\mathrm{HG}$ & down & -1.68 & FDR $<0.01$ & LOC1053 & up & 2.13 FDR $<$ \\
\hline INCO & up & 1.53 & FDR $<0.01$ & THRA1/BTR & down & -1.94 FDR $<0.01$ \\
\hline AX8- & & 1.45 & FDR $<0.01$ & LOC101C & down & -1.73 FDR $<$ \\
\hline $2 \mathrm{M}$ & wn & -1.40 & FDR $<0.01$ & CHRN & down & -1.71 FDR $<$ \\
\hline INCl & wh & -1.22 & FDR $<0.01$ & LINC & down & -1.65 FDR \\
\hline RG-AS1 & own & -1.02 & FDR $<0.01$ & 12 & down & -1.57 FDR $<1$ \\
\hline OC102724404 & down & -1.24 & FDR $<0.01$ & LINC00402 & down & -1.48 FDR $<0.01$ \\
\hline ND1-IT1 & down & -1.05 & FDR $<0.01$ & NFE4 & up & $1.42 \quad \mathrm{FDR}<0.01$ \\
\hline EmiRNA & Regulation & $\operatorname{LogFC}$ & FDR & DEmiRNA & Regulatiol & LogFC FDR \\
\hline sa-miR-494-3p & up & 2.06 & FDR $<0.01$ & hsa-miR-9-5p & up & $9.84 \quad \mathrm{FDR}<0.01$ \\
\hline sa-m & up & 1.89 & FDR $<0.01$ & hsa-miR-708-3p & & 7.37 FDR $<0.01$ \\
\hline 3p & up & 1.80 & FDR $<0.01$ & hsa-miR-1298-5p & & 7.01 FDR $<0$ \\
\hline hsa-miR-381-3p & up & 1.72 & FDR $<0.01$ & hsa-miR-379-5p & up & $6.36 \quad F D R<0.01$ \\
\hline hsa-miR-6868-3p & up & 1.72 & $\mathrm{FDR}<0.01$ & hsa-miR-136-3p & up & 6.16 FDR $<0.01$ \\
\hline hsa-miR-409-3p & up & 1.71 & FDR $<0.01$ & hsa-miR-218-5p & up & $5.92 \quad \mathrm{FDR}<0.01$ \\
\hline sa-m & up & 1.70 & FDR $<0.01$ & hsa-miR-9-3p & up & $5.80 \quad \mathrm{FDR}<0.01$ \\
\hline & & 1.66 & FDR $<0.01$ & hsa-miR-129-5p & up & $5.13 \quad \mathrm{FDR}<0.01$ \\
\hline & & 1.65 & & hsa-miR-200b-3p & up & $5.10 \quad$ FDR $<0.01$ \\
\hline hsa-miR-320b & down & 1.64 & FDR $<0.01$ & hsa-let-7e-5p & up & 4.06 FDR $<0.01$ \\
\hline
\end{tabular}




\section{Cellular Physiology Cell Physiol Biochem 2018;50:1140-1153 and Biochemistry Published online:24 October $2018 \begin{aligned} & \text { DO } 2018 \text { The Author(s). Published by S. Karger AG, Basel } \\ & \text { www.karger.com/cpb }\end{aligned}$ \\ Qian et al.: miRNA-mRNA-IncRNA Networks in COPD}

Table 3. The top 10 significantly enriched GO terms in smoking COPD

\begin{tabular}{|c|c|c|c|}
\hline GO ID & G0 name & observed gene count & false discovery rate \\
\hline \multicolumn{4}{|c|}{ Biological progress } \\
\hline G0.0070887 & cellular response to chemical stimulus & 84 & $5.36 \mathrm{E}-07$ \\
\hline G0.0042127 & regulation of cell proliferation & 59 & $1.98 \mathrm{E}-05$ \\
\hline G0.0007166 & cell surface receptor signaling pathway & 73 & $2.21 \mathrm{E}-05$ \\
\hline G0.0007154 & cell communication & 135 & $2.48 \mathrm{E}-05$ \\
\hline G0.0007155 & cell adhesion & 44 & $2.48 \mathrm{E}-05$ \\
\hline G0.0030334 & regulation of cell migration & 33 & $2.48 \mathrm{E}-05$ \\
\hline G0.0042221 & response to chemical & 109 & $2.48 \mathrm{E}-05$ \\
\hline G0.0044700 & single organism signaling & 133 & $2.48 \mathrm{E}-05$ \\
\hline G0.0071310 & cellular response to organic substance & 67 & $2.48 \mathrm{E}-05$ \\
\hline G0.0007165 & signal transduction & 126 & $3.38 \mathrm{E}-05$ \\
\hline \multicolumn{3}{|c|}{ Moulecular Function } & false discovery rate \\
\hline G0.0005515 & protein binding & 136 & 1.17E-06 \\
\hline G0.0005198 & structural molecule activity & 31 & 0.000236 \\
\hline G0.0005488 & binding & 233 & 0.000559 \\
\hline G0.0004872 & receptor activity & 50 & 0.00364 \\
\hline G0.0060089 & molecular transducer activity & 55 & 0.0184 \\
\hline G0.0003674 & molecular_function & 260 & 0.0342 \\
\hline G0.0016500 & protein-hormone receptor activity & 4 & 0.0342 \\
\hline G0.0050840 & extracellular matrix binding & 6 & 0.0342 \\
\hline G0.0008092 & cytoskeletal protein binding & 23 & 0.0354 \\
\hline G0.0005539 & glycosaminoglycan binding & 13 & 0.0366 \\
\hline \multicolumn{3}{|c|}{ Celluar compent } & false discovery rate \\
\hline G0.0005886 & plasma membrane & 139 & $1.32 \mathrm{E}-08$ \\
\hline G0.0071944 & cell periphery & 138 & $4.24 \mathrm{E}-08$ \\
\hline G0.0044459 & plasma membrane part & 83 & $2.17 \mathrm{E}-07$ \\
\hline G0.0030054 & cell junction & 49 & $2.52 \mathrm{E}-06$ \\
\hline G0.0031988 & membrane-bounded vesicle & 107 & $2.56 \mathrm{E}-06$ \\
\hline G0.0098590 & plasma membrane region & 42 & $3.49 \mathrm{E}-06$ \\
\hline G0.0016020 & membrane & 206 & 4.12E-06 \\
\hline G0.0031982 & vesicle & 108 & $4.12 \mathrm{E}-06$ \\
\hline G0.0005737 & cytoplasm & 230 & $5.91 \mathrm{E}-06$ \\
\hline G0.0044421 & extracellular region part & 110 & $5.91 \mathrm{E}-06$ \\
\hline
\end{tabular}

\section{DEIncRNA-DEmRNA interaction}

A total of 42 DElncRNA-nearby target DEmRNA pairs were obtained which were consisted of 29 DElncRNAs and 40 DEmRNAs between non-smoking COPD and normal controls. A total of 1559 DElncRNA-DEmRNA co-expression pairs were obtained which were consisted of 575 DEmRNAs and 81 DElncRNAs between non-smoking COPD and normal controls. Taken together, a total of 1601 DElncRNA-DEmRNA interaction pairs including 593 DEmRNA and 83 DElncRNAs were used to construct the non-smoking COPD-specific DElncRNA-DEmRNA interaction network (Fig. S2A). LINC01136 (degree=144), LOC105373098 (degree=126), LOC100129940 (degree=124), BISPR (degree=119) and FAM225A (degree=116) were hub DElncRNAs of this network.

A total of 23 DElncRNA-nearby target DEmRNA pairs were obtained which were consisted of 15 DElncRNAs and 19 DEmRNAs between smoking COPD and normal controls. A total of 221 DElncRNA-DEmRNA co-expression pairs were obtained which were consisted of 157 DEmRNAs and 35 DElncRNAs between smoking COPD and normal controls. Taken together, a total of 244 DElncRNA-DEmRNA interaction pairs including 208 DEmRNA and 41 DElncRNAs were used to construct the smoking COPD-specific DElncRNA-DEmRNA interaction network (Fig. S2B). LOC102724404 (degree=28), HLA-F-AS1 (degree=27), TRG-AS1 (degree=21), LINC02141 (degree=15) and PAXIP1-AS1 (degree=15) were hub DElncRNAs of this network. 


\section{Cellular Physiology Cell Physiol Biochem 2018;50:1140-1153 and Biochemistry \begin{tabular}{l|l} 
DOI: 10.1159/000494541 & $\begin{array}{l}\text { (c) } 2018 \text { The Author(s). Published by S. Karger AG, Basel } \\
\text { www.karger.com/cpb }\end{array}$
\end{tabular} \\ Qian et al.: miRNA-mRNA-IncRNA Networks in COPD}

Table 4. The top 10 significantly enriched GO terms in non-smoking COPD

\begin{tabular}{|c|c|c|c|}
\hline GO ID & GO name & $\begin{array}{c}\text { observed gene } \\
\text { count }\end{array}$ & $\begin{array}{l}\text { false discovery } \\
\text { rate }\end{array}$ \\
\hline \multicolumn{4}{|c|}{ Biological progress } \\
\hline G0.0006955 & immune response & 117 & $5.78 \mathrm{E}-17$ \\
\hline G0.0009605 & response to external stimulus & 145 & $1.10 \mathrm{E}-16$ \\
\hline G0.0002376 & immune system process & 149 & $2.75 \mathrm{E}-16$ \\
\hline G0.0006952 & defense response & 117 & $2.93 \mathrm{E}-15$ \\
\hline G0.0007166 & cell surface receptor signaling pathway & 148 & $1.13 \mathrm{E}-14$ \\
\hline G0.0051239 & $\begin{array}{l}\text { regulation of multicellular organismal } \\
\text { process }\end{array}$ & 156 & $1.27 \mathrm{E}-12$ \\
\hline G0.0002682 & regulation of immune system process & 107 & $2.42 \mathrm{E}-12$ \\
\hline G0.0070887 & cellular response to chemical stimulus & 150 & $1.12 \mathrm{E}-11$ \\
\hline G0.0042221 & response to chemical & 212 & $1.23 \mathrm{E}-11$ \\
\hline G0.0050793 & regulation of developmental process & 140 & $1.62 \mathrm{E}-11$ \\
\hline \multicolumn{4}{|c|}{ Moulecular Function } \\
\hline G0.0060089 & molecular transducer activity & 117 & $2.19 \mathrm{E}-08$ \\
\hline G0.0004871 & signal transducer activity & 105 & $1.61 \mathrm{E}-07$ \\
\hline G0.0004872 & receptor activity & 97 & $3.44 \mathrm{E}-07$ \\
\hline G0.0038023 & signaling receptor activity & 85 & $3.22 \mathrm{E}-06$ \\
\hline G0.0008201 & heparin binding & 23 & $4.96 \mathrm{E}-06$ \\
\hline G0.0005515 & protein binding & 227 & $1.86 \mathrm{E}-05$ \\
\hline G0.0003674 & molecular_function & 510 & $5.82 \mathrm{E}-05$ \\
\hline G0.1901681 & sulfur compound binding & 25 & $9.31 \mathrm{E}-05$ \\
\hline G0.0005539 & glycosaminoglycan binding & 24 & 0.000131 \\
\hline G0.0004888 & transmembrane signaling receptor activity & 73 & 0.000398 \\
\hline \multicolumn{4}{|c|}{ Celluar compent } \\
\hline G0.0071944 & cell periphery & 274 & $8.43 \mathrm{E}-18$ \\
\hline G0.0005886 & plasma membrane & 269 & $2.06 \mathrm{E}-17$ \\
\hline G0.0044459 & plasma membrane part & 153 & $3.87 \mathrm{E}-12$ \\
\hline G0.0044425 & membrane part & 306 & $3.54 \mathrm{E}-08$ \\
\hline G0.0016020 & membrane & 381 & $3.35 \mathrm{E}-07$ \\
\hline G0.0031224 & intrinsic component of membrane & 271 & 3.35E-07 \\
\hline G0.0005887 & integral component of plasma membrane & 99 & 8.29E-07 \\
\hline G0.0005615 & extracellular space & 87 & $8.52 \mathrm{E}-07$ \\
\hline G0.0044421 & extracellular region part & 195 & 8.52E-07 \\
\hline G0.0031226 & intrinsic component of plasma membrane & 101 & $9.12 \mathrm{E}-07$ \\
\hline
\end{tabular}

\section{DEmiRNA-DEmRNA interaction network}

A total of 936 DEmiRNA-DEmRNA interaction pairs including 666 DEmRNAs (151 upregulated and 515 down-regulated DEmRNAs) and 109 DEmiRNAs (81 up-regulated and 29 down-regulated DEmiRNAs) were identified in non-smoking COPD. hsa-miR-26b-5p (degree=128), hsa-miR-181d-5p (degree=110), hsa-miR-27b-3p (degree=108), hsa-miR$15 a-5 p$ (degree=106) and hsa-miR-15b-5p (degree=105) were the top 5 DEmiRNAs that owned most of target DEmRNAs in non-smoking COPD.

A total of 378 DEmiRNA-DEmRNA interaction pairs including 222 DEmRNAs (63 upregulated and 159 down-regulated DEmRNAs) and 28 DEmiRNAs (16 up-regulated and 12 down-regulated DEmiRNAs) were identified in smoking COPD. hsa-miR-30a-5p (degree $=42$ ), hsa-miR-381-3p (degree=41), hsa-miR-329-3p (degree=38), hsa-miR-452-5p (degree=32) and hsa-miR-409-3p (degree $=31$ ) were the top 5 DEmiRNAs that owned most of target DEmRNAs in smoking COPD.

\section{DEmiRNA-DEmRNA-DEIncRNA interaction network}

Non-smoking COPD-specific DElncRNA-DEmiRNA-DEmRNA interaction network was consisted of 579 nodes and 3224 edges. STRBP (degree=11), BACH2 (degree=11), SMC1A (degree=10), DDX3X (degree=9), IKZF1 (degree=9), CLCN4 (degree=9), ARSD (degree=9), 


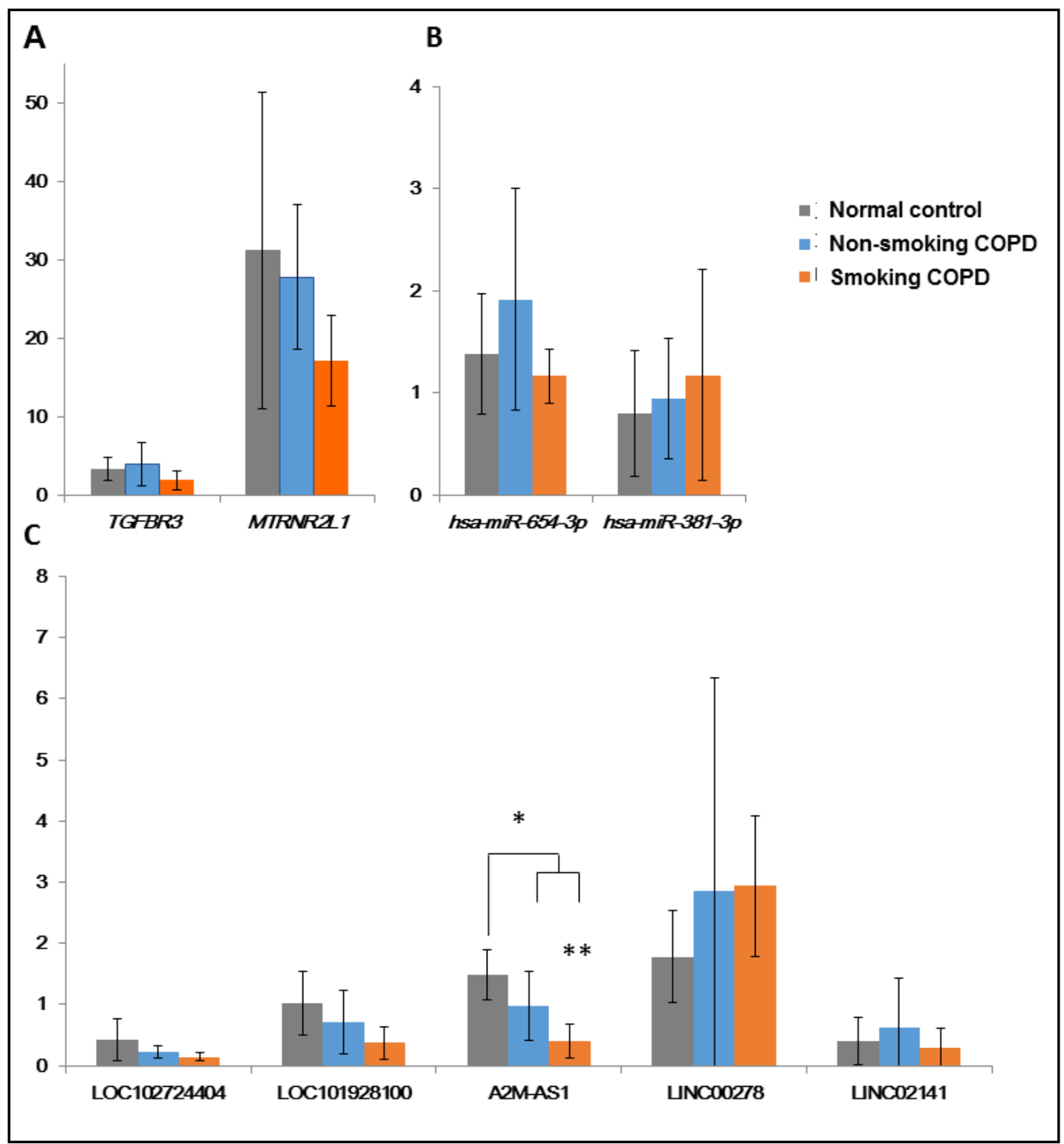

Fig. 1. Expression levels of candidate DEmRNAs, DEmiRNAs and DElncRNAs in COPD based on RNAsequencing results. The X-axis represented normal control, non-smoking COPD (Non_smo COPD) and smoking COPD (smo_COPD) group, the Y-axis represented expression levels. The names of DEmRNAs, DEmiRNAs and DElncRNAs were above the box plots.

DOK6 (degree=9) and TMTC1 (degree=9) were hub DEmRNAs of this network. MiR-218-5p/ miR15a-RORA-LOC101928100/LINC00861 interactions and miR-218-5p/miR15a-TGFß3RORA-AS1 interactions were found in non-smoking COPD-specific DElncRNA-DEmiRNADEmRNA interaction network (Fig. S3A and Fig. 1).

Smoking COPD-specific DElncRNA-DEmiRNA-DEmRNA interaction network was consisted of 187 nodes and 420 edges. RORA (degree=49), SGCD (degree=43), KLF12 (degree=43), C1orf21 (degree=39), DOK6 (degree=36), TGFBR3 (degree=33), PLXNA4 (degree=32), ATK3 (degree=31) and BCL11B (degree=30) were hub DEmRNAs of this network. Let-7-ADRB1-HLA-DQB1-AS1 interactions were found in smoking COPD-specific DElncRNA-DEmiRNA-DEmRNA interaction network (Fig. S3B and Fig. 1).

MiR-122-5p-A2M-LINC00987/A2M-AS1/linc00612 were shared interactions in both non-smoking and smoking COPD (Fig. S3C and Fig. 1). 


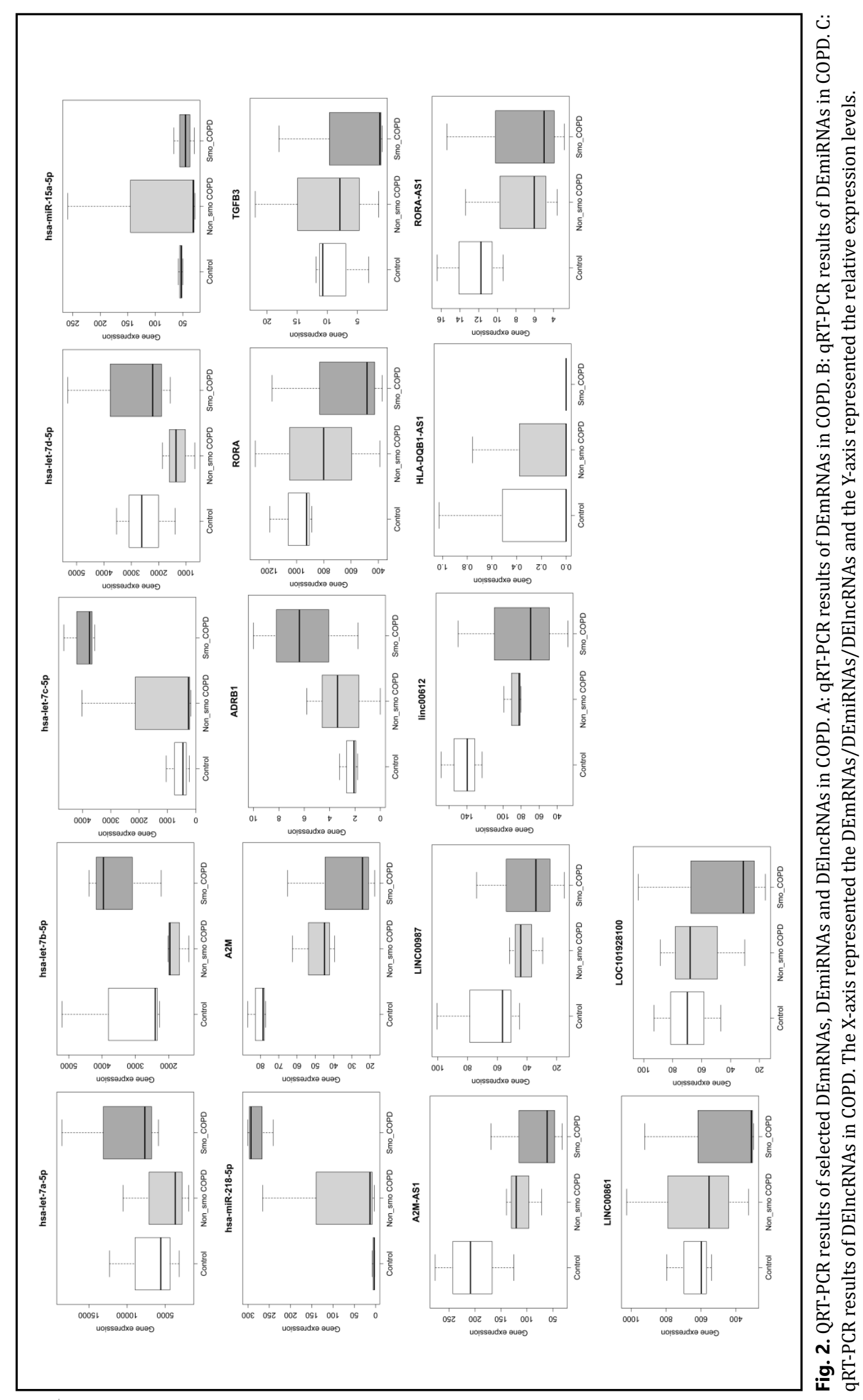




\section{Cellular Physiology Cell Physiol Biochem 2018;50:1140-1153 \begin{tabular}{ll|l} 
and Biochemistry Published online: 24 October 2018 & $\begin{array}{l}\text { (c) } 2018 \text { The Author(s). Published by S. Karger AG, Basel } \\
\text { www.karger.com/cpb }\end{array}$ \\
\hline
\end{tabular}

\section{Confirmation by $q R T-P C R$}

We performed the confirmation of two DEmRNAs (TGFB3 and MTRNR2L1), two DEmiRNAs (has-miR-654-3p and has-miR-381-3p) and five DElncRNAs (LOC102724404, LOC101928100, A2M-AS1, LINC00278 and LINC02141) by qRT-PCR. Based on our RNAsequencing results, TGFB3 and MTRNR2L1 was down-regulated in non-smoking and smoking COPD, respectively; has-miR-654-3p and has-miR-381-3p were two shared up-regulated miRNAs in both non-smoking COPD and smoking-COPD; LOC102724404 and A2M-AS1 were two shared down-regulated miRNAs in both non-smoking COPD and smoking-COPD; LOC101928100 was down-regulated while LINC00278 and LINC02141 were up-regulated in non-smoking COPD. Except for TGFB3 and has-miR-654-3p, the qRT-PCR results were consistent with our RNA-sequencing results, generally (Fig. 2).

\section{Discussion}

To better research the pathogenesis and developing potential biomarkers of nonsmoking COPD, DEmiRNAs, DEmRNAs and DElncRNAs between non-smoking patients with COPD and non-smoking controls were identified. Previous study reported that smoking COPD may has an autoimmune component which contributes to the airway inflammation in COPD even after smoking cessation [16]. Smoking-induced inflammatory and oxidative lung injury could result in production of auto-antibodies that against antigens present in tobacco or endogenous auto-antigens [17] which suggested that smoking was a potential biological foundation for the occurrence of smoking COPD. Moreover, the Global Initiative for Chronic Obstructive Lung Disease (GOLD) guidelines [18] indicated that approximately $50 \%$ of smokers eventually develop COPD. Smokers have at least a one in two chances of developing COPD if they continue smoking lifelong [19]. Hence, smoking-related genes might have a potential effect on the occurrence of COPD no matter with their expression if altered in the process of smoking COPD. In this study, we identified DEmiRNAs, DEmRNAs and DElncRNAs between smoking patients with COPD and non-smoking controls which might involve with mechanism of smoking-induced alteration including the mechanism of smoking COPD process and the potential biological foundation for occurrence of smoking COPD. Furthermore, we identified the overlapping or diverging genes, lncRNAs, miRNAs and signaling pathways in smoking and non-smoking COPD.

Based the KEGG enrichment analysis, four shared pathways including cytokinecytokine receptor interaction, ECM-receptor interaction, PI3K-Akt signaling pathway and cell adhesion molecules were identified in both smoking and non-smoking COPD. ECMreceptor interaction was reported to involve with the vascular remodeling of COPD-induced pulmonary hypertension [20]. PI3K-Akt signaling pathway was a known pathway involved with COPD as well. PI3K activation and up-regulated downstream kinase phosphorylated AKT were found in the lungs and cells of COPD patients [21]. Moreover, polymorphisms of PTEN, an inhibitor of PI3K-Akt signaling pathway were reported to be genetic risk factors for COPD[22]. Significantly increased soluble vascular cell adhesion molecule-1 (sVCAM-1) and soluble platelet endothelial cell adhesion molecular-1 (sPECAM-1) have been observed during acute exacerbation of COPD and after treatment, respectively which suggested the potential roles of cell adhesion molecules in COPD [23]. The KEGG enrichment analysis in this present study provided evidence for previous studies and suggested that these four pathways involve with COPD irrespective of smoking.

Leukocyte transendothelial migration was a significantly enriched pathway in smoking COPD. Neutrophils, a type of leukocytes was reported to play a fundamental role in the development and progression of COPD. Moreover, enhanced neutrophilendothelial interaction was reported to increase the susceptibility of smokers who develop COPD which was regulated by a1-antitrypsin [9]. Taken together, we speculated that leukocyte transendothelial migration involve with smoking COPD. Pathways in cancer and 


\section{Cellular Physiology Cell Physiol Biochem 2018;50:1140-1153

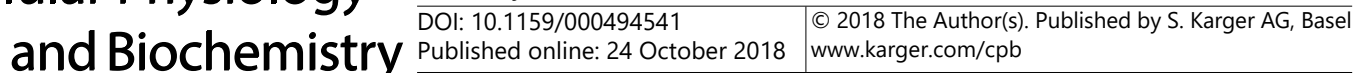 \\ Qian et al.: miRNA-mRNA-IncRNA Networks in COPD}

transcriptional misregulation in cancer were two significantly enriched pathways in nonsmoking COPD which suggested that the association between cancer and non-smoking COPD. Whether the risk for lung cancer of patients with non-smoking COPD was higher than that in patients with smoking COPD needs further research.

Both miRNAs and IncRNAs could regulate gene expression. To deeper research the biological functions of DElncRNA, DEmRNAs and DEmiRNAs in COPD, we constructed DElncRNA-DEmRNA-DEmiRNA interaction network and identified several DElncRNAmRNA-DEmiRNA interactions involved with COPD.

We identified that miR-122-5p-A2M-LINC00987/A2M-AS1/linc0061 interactions might play key roles in COPD irrespective with the smoking status. A2M, alpha 2-Macroglobulin is a major human plasma protease inhibitor that was speculated to play a role in the regulation of protease activity in the lung [24]. Dysregulation of A2M was found in smoking and nonsmoking patients with COPD compared with normal controls in both previous study [25] and this present study which suggested that A2M was a potential biomarker of COPD.

Three shared DElncRNAs in both non-smoking and smoking COPD including LINC00987, A2M-AS1 and linc00612 were all co-expressed with A2M. MiR-122-5p was a shared DEmiRNA in both non-smoking and smoking COPD that could regulate expression of A2M according to the DEmiRNA-DEmRNA interaction network in this study. Compared to normal controls, miR-122 was reported to be down-regulated in lungs of human and rats exposed to cigarette smoke [26] while was not differentially expressed in patients with COPD in previous study [27]. In this study, miR-122-5p was up-regulated in bloods of non-smoking and smoking patients with COPD. All these findings suggested that miR-122-5p might be a potential regulator of COPD. Hence, we speculated that miR-122-5p, LINC00987, A2M-AS1 and linc00612 might involve with COPD by regulating A2M.

Let-7-ADRB1-HLA-DQB1-AS1 interactions were smoking COPD-specific which might play a key role in the pathogenesis in smoking COPD. Down-regulation of let-7c has been observed in smokers with COPD compared with never smokers and the expression of let-7c was found to be associated with forced expiratory volume in 1 second $\left(\mathrm{FEV}_{1}\right)$ in previous study [28]. Moreover, let-7c was reported to be a tumor suppressor of lung cancer and serve as an important pathogenic link between COPD and lung cancer $[2,29]$. In our study, let-7a$5 p$, let-7b-5p, let-7c-5p and let-7d-5p were the top 4 down-regulated DEmiRNAs that owned most of target DEmRNAs between smoking COPD and normal controls which suggested the importance of let-7 in smoking COPD. Among shared DEmRNAs of let-7a-5p, let-7b-5p, let-7c-5p and let-7d-5p, ADRB1 (adrenoceptor beta 1) is a subtype of adrenergic receptors. Adrenergic receptors have been reported to play crucial roles in bronchomotor tone [29]. ADRB1 accounts for 30\% of adrenergic receptors in the respiratory system [29]. Moreover, the antagonists of adrenergic receptors were reported to involve with the management of exacerbated COPD [28]. In this present study, ADRB1 was up-regulated in smoking COPD compared to normal controls which emphasized its importance in smoking COPD. HLADQB1-AS1 was a co-expressed IncRNA of ADRB1. We firstly found the up-regulation of HLADQB1-AS1 in patients with smoking COPD compared to normal controls. We speculated that let-7c-ADRB1-HLA-DQB1-AS1 interactions play a key role in smoking COPD.

Among non-smoking COPD-specific DEmiRNAs in this study, miR-15a was one of the top 5 miRNAs that owned most of target DEmRNAs that up-regulated in the blood of patients with COPD compared to normal controls. Previous study also detected the overexpression of miR-15a in vastus lateralis muscle of patients with acute exacerbations of COPD [30]. Moreover, miR-15a was speculated to prevent the progression of acute exacerbations of COPD by inhibition of Wnt signaling [31].

MiR-218-5p was reported to be down-regulated in lung tissues of patients with COPD and cigarette smoke-exposed mice and indicated that miR-218-5p play a protect role in cigarette smoke-induced inflammation and COPD [32]. In this present study, miR-218-5p was up-regulated in bloods of non-smoking patients with COPD which suggested that miR218-5p might be a potential regulator of non-smoking COPD as well. 


\section{Cellular Physiology Cell Physiol Biochem 2018;50:1140-1153

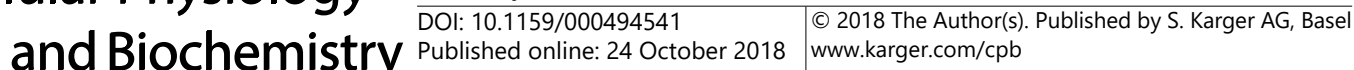 \\ Qian et al.: miRNA-mRNA-IncRNA Networks in COPD}

TGFß3 (transforming growth factor beta receptor 3) and RORA, (Retinoic acid receptor-related orphan receptor- $\alpha$ ) were two shared targets of both miR-15a and miR218-5p that were down-regulated in non-smoking patients with COPD compared to normal controls. TGF $\beta 3$ serve as a "switch" that blunted Tgfbr1/Smad2/3 and potentiated Acvrl1/Smad1 signaling in lung fibroblasts [33]. Previous studies have demonstrated the importance of TGF- $\beta$ pathway in COPD $[34,35]$. Moreover, polymorphisms of TGF $\beta 3$ have been demonstrated to be associated with the susceptibility of COPD[36]. RORA plays a key role in the regulation of lipid and cholesterol metabolism and inflammation $[37,38]$. Decreased expression of several macrophage markers (F4/80, Mac-2, Mpeg1 and Msr1) and proinflammatory cytokines (interleukin- $1 \mathrm{a},-1 \mathrm{~b}$ and -6 , and tumor necrosis factor- $\alpha$ ) were found in RORA deficient staggerer mice compared to the wild-type counterparts [39]. The inhibition of the pro-inflammatory transcription factor nuclear factor-kB RORA could be regulated by RORA as well [40]. In addition, RORA involve with the response to DNA damage, and loss of RORA protect against airspace enlargement in animal models of emphysema [41]. Moreover, variants of RORA were reported to be associated with the risk of COPD. All these findings suggested that RORA was a potential regulator of non-smoking COPD.

Two non-smoking specific DElncRNAs (LOC101928100 and LINC00861) and RORA-AS1 were co-expressed with RORA and TGF 33 , respectively. Although no previous study reported the association between COPD and these three IncRNAs, we speculated that miR-218-5p/ miR15a-RORA-LOC101928100/LINC00861 and miR-218-5p/miR15a-TGFß3-RORA-AS1 interactions play important roles in non-smoking COPD. Additionally, RORA was a nearby target gene of RORA-AS1 which suggested that RORA-AS1 might involve in COPD by its cisregulatory role on the expression of RORA.

\section{Conclusion}

In conclusion, we identified the shared and diverging genes, IncRNAs, miRNAs and signaling pathways in smoking and non-smoking COPD. Moreover, the network of lncRNAmiRNA-mRNA interactions will facilitate further experimental studies and may be used to refine biomarker predictions for developing novel therapeutic approaches in COPD. However, there are two limitations in this study. First, age and gender differences existed between the groups resulted from restrictions in sample collection. Lack of the comparison of mRNA, IncRNA and miRNA expression profiles between smoking patients with COPD and smoking controls was another limitation in this study. This comparison and further research with age-matched and sex-matched samples were needed to confirm our finding.

\section{Acknowledgements}

This work was supported by Jiangsu province "six big talent peak" project (Grant number: WSN092; Recipient: Qian Zhang), Changzhou High-Level Medical Talents Training Project (Grant number: 2016CZLJ017, Recipient: Qian Zhang), Jiangsu province "333 talents" project (Grant number: BRA2016119, Recipient: Qian Zhang) and Changzhou Sci 『Tech Program (Grant number: CJ20179031, Recipient: Qian Zhang). We thank Beijing Yangshen Bioinformatic Technology for assistance in high-throughput sequencing and data analysis.

\section{Disclosure Statement}

The authors declare that they have no conflicts of interest. 


\section{Cellular Physiology Cell Physiol Biochem 2018;50:1140-1153 \begin{tabular}{ll|l} 
and Biochemistry & Dublished online: 24 October 2018 & $\begin{array}{l}\text { (c) } 2018 \text { The Author(s). Published by S. Karger AG, Basel } \\
\text { www.karger.com/cpb }\end{array}$ \\
\hline
\end{tabular} \\ Qian et al.: miRNA-mRNA-IncRNA Networks in COPD}

\section{References}

1 Snider GL: Chronic obstructive pulmonary disease: a definition and implications of structural determinants of airflow obstruction for epidemiology. Am Rev Respir Dis 1989;140:S3-8.

-2 Murray CJ, Lopez AD: Evidence-based health policy--lessons from the Global Burden of Disease Study. Science 1996;274:740-743.

3 Salvi S: Tobacco smoking and environmental risk factors for chronic obstructive pulmonary disease. Clin Chest Med 2014;35:17-27.

4 Lamprecht B, McBurnie MA, Vollmer WM, Gudmundsson G, Welte T, Nizankowska-Mogilnicka E, Studnicka M, Bateman E, Anto JM, Burney P, Mannino DM, Buist SA: COPD in never smokers: results from the population-based burden of obstructive lung disease study. Chest 2011;139:752-763.

5 Zhou Y, Wang C, Yao W, Chen P, Kang J, Huang S, Chen B, Wang C, Ni D, Wang X, Wang D, Liu S, Lu J, Zheng J, Zhong N, Ran P: COPD in Chinese nonsmokers. Eur Respir J 2009;33:509-518.

6 Lamprecht B, Schirnhofer L, Kaiser B, Buist S, Studnicka M: Non-reversible airway obstruction in never smokers: results from the Austrian BOLD study. Respir Med 2008;102:1833-1838.

7 Zeng G, Sun B, Zhong N: Non-smoking-related chronic obstructive pulmonary disease: a neglected entity? Respirology 2012;17:908-912.

8 De Smet EG, Mestdagh P, Vandesompele J, Brusselle GG, Bracke KR: Non-coding RNAs in the pathogenesis of COPD. Thorax 2015;70:782-791.

-9 Gu C, Li Y, Liu J, Ying X, Liu Y, Yan J, Chen C, Zhou H, Cao L, Ma Y: LncRNAmediated SIRT1/FoxO3a and SIRT1/p53 signaling pathways regulate type II alveolar epithelial cell senescence in patients with chronic obstructive pulmonary disease. Mol Med Rep 2017;15:3129-3134.

10 Bartel DP: MicroRNAs: genomics, biogenesis, mechanism, and function. Cell 2004;116:281-297.

11 Vadaie N, Morris KV: Long antisense non-coding RNAs and the epigenetic regulation of gene expression. Biomol Concepts 2013;4:411-415.

12 Zhang C, Wang P, Jiang P, Lv Y, Dong C, Dai X, Tan L, Wang Z: Upregulation of IncRNA HOTAIR contributes to IL-1beta-induced MMP overexpression and chondrocytes apoptosis in temporomandibular joint osteoarthritis. Gene 2016;586:248-253.

13 Afonso AS, Verhamme KM, Sturkenboom MC, Brusselle GG: COPD in the general population: prevalence, incidence and survival. Respir Med 2011;105:1872-1884.

14 Shaikh M, Sood RG, Sarkar M, Thakur V: Quantitative Computed Tomography (CT) Assessment of Emphysema in Patients with Severe Chronic Obstructive Pulmonary Disease (COPD) and its Correlation with Age, Sex, Pulmonary Function Tests, BMI, Smoking, and Biomass Exposure. Pol J Radiol 2017;82:760766.

15 Bonasio R, Shiekhattar R: Regulation of transcription by long noncoding RNAs. Annu Rev Genet 2014;48:433-455.

16 Agusti A, MacNee W, Donaldson K, Cosio M: Hypothesis: does COPD have an autoimmune component? Thorax 2003;58:832-834.

17 Cosio MG: Autoimmunity, T-cells and STAT-4 in the pathogenesis of chronic obstructive pulmonary disease. Eur Respir J 2004;24:3-5.

-18 Gomez FP, Rodriguez-Roisin R: Global Initiative for Chronic Obstructive Lung Disease (GOLD) guidelines for chronic obstructive pulmonary disease. Curr Opin Pulm Med 2002;8:81-86.

19 Lundback B, Lindberg A, Lindstrom M, Ronmark E, Jonsson AC, Jonsson E, Larsson LG, Andersson S, Sandstrom T, Larsson K: Not 15 but 50\% of smokers develop COPD?--Report from the Obstructive Lung Disease in Northern Sweden Studies. Respir Med 2003;97:115-122.

20 Hoffmann J, Wilhelm J, Marsh LM, Ghanim B, Klepetko W, Kovacs G, Olschewski H, Olschewski A, Kwapiszewska G: Distinct differences in gene expression patterns in pulmonary arteries of patients with chronic obstructive pulmonary disease and idiopathic pulmonary fibrosis with pulmonary hypertension. Am J Respir Crit Care Med 2014;190:98-111.

21 To Y, Ito K, Kizawa Y, Failla M, Ito M, Kusama T, Elliott WM, Hogg JC, Adcock IM, Barnes PJ: Targeting phosphoinositide-3-kinase-delta with theophylline reverses corticosteroid insensitivity in chronic obstructive pulmonary disease. Am J Respir Crit Care Med 2010;182:897-904.

-22 Hosgood HD, 3rd, Menashe I, He X, Chanock S, Lan Q: PTEN identified as important risk factor of chronic obstructive pulmonary disease. Respir Med 2009;103:1866-1870. 


\section{Cellular Physiology Cell Physiol Biochem 2018;50:1140-1153 \begin{tabular}{ll|l} 
DOI: 10.1159/000494541 & $\begin{array}{l}\text { O 2018 The Author(s). Published by S. Karger AG, Basel } \\
\text { www.karger.com/cpb }\end{array}$ \\
\hline
\end{tabular} \\ Qian et al.: miRNA-mRNA-IncRNA Networks in COPD}

23 Pan L, Yang YH, Dong DQ Liu XC: [Detection and clinical significance of vascular cell adhesion molecules-1 in chronic obstructive pulmonary disease]. Zhonghua Jie He He Hu Xi Za Zhi 2008;31:291-293.

-24 Poller W, Barth J, Voss B: Detection of an alteration of the alpha 2-macroglobulin gene in a patient with chronic lung disease and serum alpha 2-macroglobulin deficiency. Hum Genet 1989;83:93-96.

-25 Arellano-Orden E, Calero-Acuna C, Cordero JA, Abad-Arranz M, Sanchez-Lopez V, Marquez-Martin E, Ortega-Ruiz F, Lopez-Campos JL: Specific networks of plasma acute phase reactants are associated with the severity of chronic obstructive pulmonary disease: a case-control study. Int J Med Sci 2017;14:67-74.

-26 Izzotti A, Calin GA, Arrigo P, Steele VE, Croce CM, De Flora S: Downregulation of microRNA expression in the lungs of rats exposed to cigarette smoke. Faseb J 2009;23:806-812.

-27 Donaldson A, Natanek SA, Lewis A, Man WD, Hopkinson NS, Polkey MI, Kemp PR: Increased skeletal muscle-specific microRNA in the blood of patients with COPD. Thorax 2013;68:1140-1149.

28 Antonelli-Incalzi R, Pedone C: Respiratory effects of beta-adrenergic receptor blockers. Curr Med Chem 2007;14:1121-1128.

29 Carstairs JR, Nimmo AJ, Barnes PJ: Autoradiographic visualization of beta-adrenoceptor subtypes in human lung. Am Rev Respir Dis 1985;132:541-547.

-30 Duan Y, Zhou M, Xiao J, Wu C, Zhou L, Zhou F, Du C, Song Y: Prediction of key genes and miRNAs responsible for loss of muscle force in patients during an acute exacerbation of chronic obstructive pulmonary disease. Int J Mol Med 2016;38:1450-1462.

31 Reuter S, Beckert H, Taube C: Take the Wnt out of the inflammatory sails: modulatory effects of Wnt in airway diseases. Lab Invest 2016;96:177-185.

-32 Conickx G, Mestdagh P, Avila Cobos F, Verhamme FM, Maes T, Vanaudenaerde BM, Seys LJ, Lahousse L, Kim RY, Hsu AC, Wark PA, Hansbro PM, Joos GF, Vandesompele J, Bracke KR: MicroRNA Profiling Reveals a Role for MicroRNA-218-5p in the Pathogenesis of Chronic Obstructive Pulmonary Disease. Am J Respir Crit Care Med 2017;195:43-56.

-33 Zhong N, Wang C, Yao W, Chen P, Kang J, Huang S, Chen B, Wang C, Ni D, Zhou Y, Liu S, Wang X, Wang D, Lu J, Zheng J, Ran P: Prevalence of chronic obstructive pulmonary disease in China: a large, population-based survey. Am J Respir Crit Care Med 2007;176:753-760.

-34 de Boer WI, van Schadewijk A, Sont JK, Sharma HS, Stolk J, Hiemstra PS, van Krieken JH: Transforming growth factor beta1 and recruitment of macrophages and mast cells in airways in chronic obstructive pulmonary disease. Am J Respir Crit Care Med 1998;158:1951-1957.

35 Morris DG, Huang X, Kaminski N, Wang Y, Shapiro SD, Dolganov G, Glick A, Sheppard D: Loss of integrin alpha(v)beta6-mediated TGF-beta activation causes Mmp12-dependent emphysema. Nature 2003;422:169-173.

-36 Hersh CP, Hansel NN, Barnes KC, Lomas DA, Pillai SG, Coxson HO, Mathias RA, Rafaels NM, Wise RA, Connett JE: Transforming growth factor-beta receptor-3 is associated with pulmonary emphysema. Am J Respir Cell Mol Biol 2009;41:324.

-37 Barnes PJ: New anti-inflammatory targets for chronic obstructive pulmonary disease. Nat Rev Drug Discov 2013;12:543-559.

-38 Kolsum U, Roy K, Starkey C, Borrill Z, Truman N, Vestbo J, Singh D: The repeatability of interleukin-6, tumor necrosis factor-alpha, and C-reactive protein in COPD patients over one year. Int J Chron Obstruct Pulmon Dis 2009;4:149-156.

-39 Kang HS, Okamoto K, Takeda Y, Beak JY, Gerrish K, Bortner CD, DeGraff LM, Wada T, Xie W, Jetten AM: Transcriptional profiling reveals a role for RORalpha in regulating gene expression in obesity-associated inflammation and hepatic steatosis. Physiol Genomics 2011;43:818-828.

-40 Delerive P, Monte D, Dubois G, Trottein F, Fruchart-Najib J, Mariani J, Fruchart JC, Staels B: The orphan nuclear receptor ROR alpha is a negative regulator of the inflammatory response. EMBO Rep 2001;2:42-48.

41 Shi Y, Cao J, Gao J, Zheng L, Goodwin A, An CH, Patel A, Lee JS, Duncan SR, Kaminski N, Pandit KV, Rosas IO, Choi AM, Morse D: Retinoic acid-related orphan receptor-alpha is induced in the setting of DNA damage and promotes pulmonary emphysema. Am J Respir Crit Care Med 2012;186:412-419. 